\title{
Effects of adult familiarity on social behaviours in Angelman syndrome.
}

Mount, R., Oliver, C., Berg, K. and Horsler, K.

Cerebra Centre for Neurodevelopmental Disorders, School of Psychology, University of Birmingham

Please use this reference when citing this work:

Mount, R., Oliver, C., Berg, K. and Horsler, K. (2011). Effects of adult familiarity on social behaviours in Angelman syndrome. Journal of Intellectual Disability Research, 55, 339-350. doi: 10.1111/j.13652788.2010.01364.x

The Cerebra Centre for Neurodevelopmental Disorders,

School of Psychology, University of Birmingham, Edgbaston, Birmingham, B15 2TT

Website: www.cndd.Bham.ac.uk

E-mail: cndd-enquiries@contacts.bham.ac.uk 


\section{Effects of adult familiarity on social behaviours in Angelman syndrome}

Keywords: Angelman syndrome, behavioural phenotype, imprinting, kinship theory, sociability, intellectual disability 


\begin{abstract}
Background. Individuals with Angelman syndrome appear strongly motivated by social contact but there have been few studies which have examined the relationship between sociability and familiarity. In this study we compared social behaviour in Angelman syndrome when in contact with mothers and strangers.
\end{abstract}

Methods. We systematically manipulated adult familiarity, eye contact and speech to examine the effect on social approach behaviours in children with Angelman syndrome. Eleven children (deletion 15q11-13) participated and were observed during interactions with their mother and an unfamiliar adult while adult eye contact and talking were manipulated. Laughing and smiling, looking and social approach were observed.

Results. There was no effect of familiarity on laughing and smiling or eye contact. Participants showed more social approach towards their mother than the unfamiliar adult but only when their mother was looking at them.

Conclusions. In Angelman syndrome, looking at adults, laughing and smiling appear to be unaffected by the familiarity of the adult. However, approach behaviours are more common with mothers than strangers. The function of the approach behaviours might be to increase investment from the primary caregiver. 


\section{Introduction}

Angelman syndrome is caused by disruption to the maternally inherited portion of chromosome 15q11-13 and affects between 1:10,000 and 1: 40,000 live births (ClaytonSmith, 1993). Approximately $70 \%$ of people with Angelman syndrome have a deletion on the maternally derived chromosome $15 q 11-13,2-7 \%$ have unipaternal disomy, $2-7 \%$ have an imprinting defect and 5-15\% have a mutation in the UBE3A gene (Jiang et al., 1999). Between $5-10 \%$ of people who show the clinical features have no demonstrable cytogenetic or molecular abnormality of chromosome 15q11-13 (Lossie et al., 2001). Physical features include movement disorder (Williams et al., 1995), microcephaly, epilepsy and abnormal EEG (Williams et al., 1995; Zori et al., 1992), prognathia, strabismus, hypopigmented skin, hair and eye colour (Williams et al., 1995) and dysmorphic facial features (Angelman, 1965). Overall, individuals have severe levels of intellectual disability and severe speech impairment (Berg \& Pakula, 1972; Andersen, Rasmussen, \& Stromme, 2001; In review citation withheld for blind review).

A number of behavioural features have been reported and the most prominent are 'excessive' is laughing and smiling (Horsler \& Oliver, 2006a; In review citation withheld for blind review). These behaviours are common (seen in over $80 \%$, Summers, Allison, Lynch, \& Sandler, 1995; Williams et al., 1995;) and frequently shown (e.g. Fridman, Varela, Kok, Diament, \& Koiffmann, 2000; Sandanam et al., 1997). Whether or not these behaviours are inappropriate has been the subject of debate. Early reports conceptualised laughing and smiling as unrelated to environment context (e.g. Angelman, 1965, Dooley, Berg, Pakula, \& MacGregor, 1981, Williams \& Frias, 1982). More recently Oliver, Demetriades, and Hall (2002) showed that higher levels of laughing and smiling were elicited during adult interaction than when the children were alone. Richman, Gernach and Teichman (2006) did not replicate this finding but their sample included younger children. Horsler and Oliver (2006b) have subsequently demonstrated higher frequency of laughing and smiling when adult speech, touching, smiling, laughing and eye contact were ongoing compared to adult speech only and when the adult just sat adjacent to the child. In a further study Oliver, Horsler, Berg et al. (2007) showed the amount of time children with Angelman syndrome engaged in laughing and smiling was higher than for a matched comparison group and higher when social contact was ongoing. In combination these studies provide evidence that laughing and smiling behaviours are phenotypic for Angelman syndrome, can be related to social influences and thus unlikely to be a randomly occurring phenomenon. 
Throughout the Angelman syndrome case reports, descriptions of laughing and smiling behaviour are often accompanied by reports of an excitable personality and a positive demeanour (e.g. Fridman et al., 2000; Moncla et al., 1999a; Saitoh et al., 1997; Williams et al., 1995, Horsler and Oliver, 2006b). However, details on how these features manifest themselves are not provided. Findings from studies profiling behavioural, emotional and personality characteristics suggest people with Angelman syndrome may be more sociable than others with intellectual disabilities. Summers and Feldman (1999) compared the behavioural and emotional features of 27 individuals with Angelman syndrome to those seen in a community and clinic sample of individuals with intellectual disabilities. Individuals with Angelman syndrome scored at a lower level on the irritability and lethargy subscales of the Aberrant Behavior Checklist (ABC, Aman \& Singh, 1986) with fewer temper tantrums and less withdrawal. Similar findings were reported by Clarke and Marston (2000) who also used the $A B C$ to examine behavioural difficulties in 73 individuals with $A S$. They found the Angelman syndrome group showed fewer behaviours related to lethargy and withdrawal than a group of 34 individuals with Prader-Willi syndrome and 26 individuals with Smith-Magenis syndrome.

Studies have also assessed the profile of social and communication skills in AS. Peters et al., 2004 used the Vineland Adaptive Behavior Scales (VABS, Sparrow, Balla, \& Cicchetti, 1984 ) to assess nineteen children with Angelman syndrome. The socialisation domain of the VABS assesses purposeful interactive skills and this was found to be a strength compared to communication and daily living skills. A similar finding was reported by Jolleff and Ryan (1993) who investigated verbal and non-verbal communication behaviours in eleven children with Angelman syndrome. They found that all children could communicate their needs, and seven children showed evidence of social interaction without communication. However, these studies did not provide any details on how social interactions with others were initiated or maintained by children with Angelman syndrome.

Some of the behavioural difficulties found to be associated with Angelman syndrome could be conceptualised as excessive or disordered social interaction. Clarke et al. (2000) found the behaviours causing most concern to parents included disturbing others, being dependent and attention seeking. Summers et al. (1995) found $91 \%$ of 11 individuals studied were described as attention seeking by their parents and all were reported to show aggressive behaviour. This aggressive behaviour was related to grabbing people or things rather than to acts of physical aggression. Recently (citation withheld for blind review) have shown 
aggression in children and adults to be over three times more likely to occur in AS than in a contrast group. Clayton-Smith (2001) in a study of 28 adults with Angelman syndrome, commented that a cause of aggression could be an inability to get needs met, as those with better communication skills seemed to have less aggression problems.

Although there are many limitations, the literature published so far on the social behaviour in Angelman syndrome suggests individuals laugh and smile in relation to social manipulations, present with less anti-social and withdrawal behaviours than others with intellectual disabilities, show strengths in socialisation compared to communication skills and show a range of "difficult" behaviours, some of which appear to be socially motivated. However, to date there are no studies that consider whether the smiling and laughing and the social approach behaviours differ when in the presence of primary caregivers as opposed to, for example, an unfamiliar adult. This type of comparison would be informative with regard to other effects on the behaviour of children with Angelman syndrome.

In this study we assess empirically social behaviour in Angelman syndrome using observational methods. Previous experimental studies of laughing and smiling have indicated that adult eye contact, laughing and smiling, talking and touching are evocative of laughing and smiling in Angelman syndrome (Oliver et al., 2002; Horsler \& Oliver, 2006; Oliver, Horsler, Berg, et al. 2007). However, many factors were combined across conditions and so these studies could not provide information of which aspects of social interaction were most influential. In this study we manipulate three aspects of dyadic social interaction. Familiarity of the adult is manipulated to compare social interaction with mother compared to a female stranger. Adult eye contact and talking are manipulated to determine whether eye contact and talking are particularly evocative aspects of social interaction. To gain a more complete picture of the social behaviour than that previously described, several behaviours shown by individuals with Angelman syndrome were recorded: looking at their interactive partner, laughing and smiling, a number of non-aggressive (reaching, touching) and aggressive (pulling) approach behaviours. The adults were instructed to try not to laugh or smile during the interactions. This provided the possibility of observing whether individuals with Angelman syndrome laugh and smile in the absence of others showing these behaviours. Due to the difficulty adults experienced in suppressing laughing and smiling conditions were limited to 15 seconds in duration. This was also essential given the high levels of distractability shown by participants that would have compromised the integrity of the experimental conditions. The hypothesis of the study is the amount of laughing/smiling, looking, reaching, touching 
and pulling shown by individuals with Angelman syndrome will vary with the three aspects of social interaction manipulated (adult familiarity, eye contact and talking).

\section{Method}

\section{Participants}

Fifteen individuals with Angelman syndrome were recruited via the Angelman Syndrome Support Education Research Trust (ASSERT) in the UK from an existing database of 125 children and adults with AS. Seventeen families who were within a reasonable travelling distance agreed to take part. Two were subsequently excluded due to travel constraints. Table 1 presents data on the gender, age, genetic cause and adaptive behaviour of the participants. Adaptive abilities were assessed using the adaptive composite from the Vineland Adaptive Behavior Scales (Sparrow et al., 1984). Experimental observations were not completed for two individuals. These were P4 (female, age 4.7 years) and P14 (female, age 18.5 years). Both of these participants were mobile, highly active and distractible. During observations they wandered around the room rather than sitting and interacting with the adult. It is noteworthy that these two participants did not have a 15q11-13 deletion.

Observational data were available for 13 participants ( 8 males, 5 females). The fathers of two participants acted as the familiar adult throughout the observations (P5, female, age 5.3 years and $\mathrm{P} 13$, male, age 12.1 years). The hypothesis of the study relates to interaction with the primary care giver, which was the mother for these participants, and so these participants were excluded from analyses leaving a sample size of 11 ( 7 males, 4 females). The mean age was 7.8 years $(S D=4.3$, range 3 - 18.5 years). The mean age equivalent adaptive level was 13.1 months ( $S D=4.1$, range 8 - 20.7 months). Two participants were classified as having profound adaptive deficits, six had severe and three had moderate deficits.

++++++++++ Insert table 1 here ++++++++++

\section{Measures}

Behavioural recordings. Observers collected data on independent and dependent variables using the Obswin32 software (Martin, Oliver, \& Hall, 2002). It allows the occurrence of 
behaviours to be simultaneously recorded in continuous time and so avoids the need for any form of time sampling.

\section{Procedure}

One visit was conducted at a participant's school; all other visits were conducted in participants' homes. During the visit, a member of the research team administered the VABS and conducted the experimental observations. The observations were conducted in a quiet room with minimal distractions. Two adults and the participant were present.

The design of the study was factorial with three factors (adult familiarity, eye contact and talking), each with two levels (familiar vs. unfamiliar, eye contact vs. no eye contact and talking vs. no talking). These factors were combined to produce four experimental conditions to be conducted with the familiar and unfamiliar adult. Table 2 presents descriptions of the conditions. Each participant was exposed to four conditions in four trials with their mother, and four trials with an unfamiliar adult. For five of the participants, the trials with their mother occurred first. For the other six participants the trials with the unfamiliar adult occurred first. The unfamiliar adult was the same for all participants and was a female member of the research team (RM).

The order in which the four conditions were presented within each of the trials was randomised and determined prior to the visit using a random sequence generator (Haahr, 2002). In all situations the adult sat adjacent to the participant, facing their body towards them. Before each condition began the adult gained the participant's attention by calling their name. Each condition was presented for 15 seconds. All observations were video recorded for later analysis. The unfamiliar and familiar adult operated the video recorder.

++++++++ Insert Table 2 here ++++++++++++

\section{Behavioural recording and interobserver reliability}

To evaluate the integrity of two of the independent variables, adult eye contact and adult talking were recorded. Adult touching and adult laughing/smiling were also recorded to assess whether they occurred despite the adults being instructed not to show the behaviours. Definitions for the adult behaviours are presented in Table 3. Seven participant behaviours were recorded as dependent variables: looking at the adult, laughing/smiling, 
reaching for the adult, touching adult head, touching adult body, pulling adult head, pulling adult body. Looking at the camera was included as an indication of how aware the participants were of the camera. Definitions of these behaviours are shown in Table 3.

Interobserver agreement was assessed by a second observer simultaneously but independently recoding behaviour in approximately $25 \%$ of all the 15 -second sessions in which the behaviours occurred ( 279 sessions were coded twice out of a total of 1100). The sample of $25 \%$ included sessions from all conditions and all participants. Kappa indices were calculated based on a one-second interval-by-interval comparison of observer records. The Kappa coefficients for the behaviours are shown in Table 3. Indices for all variables were greater .50 indicating acceptable levels of reliability (Landis \& Koch, 1977).

\section{Data analysis}

Five of the participant behaviours (reaching toward, pulling and touching adult head or body) occurred at a low duration (less than $12 \%$ of the time across conditions, range for behaviours $1.38 \%$ to $11.46 \%$ ). Separate analyses of these five behaviours were not feasible due to their low duration. All five behaviours involved the participant reaching out in some way towards the adult and thus part of the same response class of behaviour, all potentially serving a similar function (Leslie, 2002). Consequently, these behaviours were combined and analysed as approach behaviours.

Three repeated measures, within-subject ANOVAs were used to evaluate the hypothesis. One-sample Kolmogrov-Smirnov tests confirmed the data for participant laughing/smiling, eye contact and approach were normally distributed. Participant laughing/smiling, looking and approach were dependent variables. The independent variables were entered as three within-subject factors with two levels (Familiarity = familiar vs. unfamiliar, Talk = talking vs. no talking, and Eye contact = eye contact vs. no eye contact). To determine the direction of any significant interaction effects, post-hoc tests were completed (Tukey's HSD tests), which take account of the risk of making Type I errors due to multiple comparisons.

\section{Results}

To determine the integrity of the conditions the mean percentage occurrences of adult eye contact and adult talking were examined across conditions. The percentage of intervals in which adult eye contact occurred was close to $100 \%$ in the four eye contact conditions 
(range $97.16 \%$ to $98.96 \%$ ) and close to $0 \%$ in the four no eye contact conditions (range $.71 \%$ to $3.55 \%$ ). The percentage of intervals in which adult talking occurred was close to $100 \%$ in the four talking conditions (range $95.6 \%$ to $100 \%$ ) and close to $0 \%$ in the four no talking conditions (range $0 \%$ to $.71 \%$ ).

To determine presence of other adult behaviours, the mean percentage of adult laughing/smiling and touching were examined across conditions (see Table 4). The means presented in Table 4 suggest there was more adult laugh/smile in the eye contact and talking conditions. These observations were confirmed by a repeated measures, within-subject ANOVA. There was a significant main effect of eye contact (Wilk's Lambda $=.51, f=9.54$, $\mathrm{df}=10, \mathrm{p}=.011$ ), and talk (Wilk's Lambda=.65, $\mathrm{f}=5.33$, $\mathrm{df}=10, \mathrm{p}=.044$ ) but no significant interaction effects. The means in Table 4 suggest a similar pattern for adult touch. A repeated measures within-subject ANOVA with adult touch entered as the dependent variable and familiarity, eye contact and talking as independent variables confirmed there was a significant main effect of eye contact (Wilk's Lambda=.66, $f=5.05, d f=10, p=.048$ ), but not for talk and there were no significant interaction effects.

+++++++++++ Insert Table 4 here +++++++++++

To examine the frequency of participant behaviours and participant variability, the percentage of intervals each participant showed the behaviours across all the experimental sessions was calculated (see Table 5). Table 5 highlights considerable variability between participants. Greatest variability was seen for laugh/smile. P3 laughed/smiled in only $6 \%$ of intervals, whereas P15 laughed/smiled in 75\%. Variability was also seen for approach behaviours. P8 made approaches to the adults in $9 \%$ of the intervals whereas P2 made approaches in $43 \%$. Looking at the adult was a frequent behaviour; the lowest percentage was shown by P6 (25\%). Looking at the camera was a comparatively infrequent behaviour; the highest frequency was shown by P15 (24\%, range $5.03 \%$ to $24.02 \%)$.

++++++++++ Insert Table 5 here +++++++++++

Figure 1 presents the mean duration of participant laugh/smile, looking and approach behaviours across conditions. Three repeated measures, within-subject ANOVAs were conducted to evaluate the main hypothesis of the study.

++++++++++++ Insert Figure 1 here ++++++++++++ 
Results of the Analyses of Variance showed there were no significant effects of the factors for participant laugh/smile and eye contact. There was, however, a trend toward significance $(F(10,1)=4.39, p=.063)$ for a second order interaction between familiarity, eye contact and talking with the highest level being observed in the condition in which the mother was maintaining eye contact and talking with her child. There were no significant main or interaction effects for the outcome variable participant looking. For participant approach behaviours there was a significant main effect of familiarity $(F(10,1)=7.84, p=.019)$ and a significant familiarity by eye contact interaction effect $(F(10,1)=7.03, p=.024)$. To determine the direction of the interaction, post-hoc Tukey's HSD tests were conducted for the six possible combinations of the conditions. Just one significant difference was found. Significantly more approach behaviour was shown when the familiar adult engaged in eye contact (marginal mean=44.25) compared to when the unfamiliar adult engaged in eye contact (marginal mean=18.74) $(p=.032)$. No other effects reached statistical significance, although the interaction between familiarity and looking approached significance $(F(10,1)=4.95, p=.05)$. Thus, the difference between the familiar and unfamiliar adults when they maintained eye contact with the participants accounted for the main effect of familiarity.

To investigate factors that may contribute to variability seen between participants, Pearson Product Moment Correlation Coefficients were calculated between chronological age, age equivalent adaptive level from the VABS and participant laughing/smiling, looking and approach behaviour. There was a significant positive association between adaptive abilities and participant laughing/smiling $(r(10)=.75, p=.009)$ and between adaptive abilities and participant looking $(r(10)=.72, p=.012)$. No other significant correlations were evident.

\section{Discussion}

This is the first study to look at the effect of adult familiarity on a range of prosocial behaviours evident in Angelman syndrome using an experimental approach The design provided control over three aspects of interaction (adult familiarity, adult eye contact and talking). A high level of reliability was found for the behaviours recorded and integrity of the conditions was demonstrated. The study evaluated the hypothesis that laughing/smiling, looking, reaching, touching and pulling shown by individuals with Angelman syndrome would vary with the three aspects of social interaction manipulated. 
Familiarity of the adult was manipulated to compare social interaction with the mother compared to a female stranger. The findings from the current study related to approach behaviour show higher levels of approach behaviour directed toward the mother. It was not possible to assess the effects of the adult manipulations on the five reaching, touching and pulling behaviours separately. These behaviours were shown in low frequency by many of the participants. The behaviours were all part of a response class of approach behaviours with the likely function of gaining or maintaining contact from others and so were combined. The participants showed more approach behaviour when they were interacting with the familiar compared to the unfamiliar adult but only when the adults were maintaining eye contact. Interestingly, it was not the case that the individuals consistently showed more approaches towards their mother compared to the strangers across all conditions. The fact that the individuals showed more approaches towards their mother only when the mother was maintaining eye contact suggests the individuals with Angelman syndrome were more motivated to maintain eye contact with their mother than a stranger once it was provided. However, they were no more likely to attempt to gain the eye contact of their mother over that of a stranger. The findings suggest that one of the functions of the approach behaviours is to maintain eye contact with their mother.

The results showed a trend for participants laughing and smiling more when their mother was talking to and maintaining eye contact with them compared to other conditions. However, this effect failed to reach statistical significance $(p=.063)$. The effects for familiarity and talk alone were far from reaching statistical significance $(p=.872$ and .661 respectively) although the effect for eye contact approached significance (.053). The direction of this effect is consistent with the findings of Horsler and Oliver (2006b) who found more laughing and smiling in a condition with adult speech, eye contact, laughing, smiling and touching compared to a condition with just adult speech.

The amount the participants looked at the adults during the observations was frequent $(>30 \%$ of intervals) but did not vary across conditions. The findings suggest that the individuals with Angelman syndrome looked at the adult regardless of whether they were talking, looking at them or whether they were a familiar or strange person. The high degree of interest individuals with Angelman syndrome show in adults is one factor that might have contributed to the descriptions of sociability found previous reports (e.g. Clarke et al., 2000; Summers et al., 1999 ; Oliver, Horsler, Berg et al., 2007). 
Considerable variability was seen across participants. Adaptive abilities were found to be positively associated with the amount of laughing/smiling and looking, with more able participants showing a higher frequency of these behaviours. Interestingly, no association was found between adaptive abilities and the amount of approach behaviour. Factors contributing to variability in the Angelman syndrome phenotype are not well researched. One factor found to contribute to variability is genotype with a milder clinical presentation being seen in individuals without a deletion (Fridman et al., 2000; Lossie et al., 2001; Moncla et al., 1999b). All participants that took part in the observations had deletions in 15q11-13. However, the two participants who did not sit to interact with the adult during the observations did not have deletions (imprinting defect and UPD). From the small number of individuals who took part in this study, it seemed the degree of intellectual disability was milder in those without deletions. Due to the way the experiment was designed, it was not possible to capture the social behaviour of the individuals without deletions. Future research might explore methods of capturing the variability in social behaviour across the different genetic subtypes.

The study has a number of limitations to evaluate the hypothesis. The sample size was small which may have contributed to a lack of power to find key effects such as association between familiarity, adult eye contact and participant laughing/smiling, which only approached statistical significance. The sample may not be entirely representative of Angelman syndrome because participants were recruited via the Angelman syndrome support organisation. This could have introduced a possible sampling bias. The conditions were presented for just 15 seconds. Longer conditions would have allowed for closer examination of the functions of the behaviours, particularly the reaching, touching and pulling behaviours. If these behaviours are part of the response class of approach behaviours, with a function of gaining or maintaining eye contact, an escalation from milder forms of the behaviour (e.g. reaching) to more aggressive forms of the behaviour (e.g. pulling) in the conditions where eye contact was not provided might have been evident as predicted by the response class hierarchy hypothesis (Richman, Wacker, Asmus, Casey, \& Andelman, 1999; Lalli, Mace, Wohn, \& Livezey, 1995).

Although the integrity of the conditions regarding adult talking and eye contact was robust, there was significant variability in amount of adult laugh/smile and touch across conditions. It is unlikely that these variations were responsible for the significant familiarity by eye contact interaction effect found for participant approach behaviours, as there were no significant 
differences in the amount of laugh/smile and touch shown by the familiar and unfamiliar adults. It is interesting that both the familiar and unfamiliar adults laughed and smiled despite being instructed not to. Horsler and Oliver (2006b) found an association between child laughing and smiling and adult laughing and smiling but were not able to determine whether the child behaviour had elicited the adult behaviour or vice versa. Oliver et al. (2007) subsequently used lag sequential analysis to show that in the natural environment children with Angelman syndrome approached adults, smiled and then the adults smiled. In this study, the fact that the adults laughed and smiled despite being instructed not to suggests that the child behaviours were eliciting the response from the adults in a similar way. Finally, it would be beneficial to replicate this experiment using an appropriately matched contrast group to evaluate the strength of the differentiation between familiar and unfamiliar adults in Angelman sydnrome

Despite the limitations, the study provides evidence that the repertoire of social behaviour in individuals with Angelman syndrome is more extensive than functional laughing and smiling behaviour and also includes looking and approach behaviours. The results indicate that adult eye contact and familiarity are important aspects of dyadic social interaction for people with AS. The study deliberately excluded adult laughing/smiling from the conditions to avoid confounding laughing and smiling in the child with AS as a response to the adults' behaviour. However, the fact that the adults did laugh and smile indicates this is a natural response to these behaviours that is difficult to suppress.

Findings from previous studies suggested that one function of aggressive behaviour shown by children and adults with Angelman syndrome might be to gain the attention of others (Clayton-Smith, 2001; Summers et al., 1995; Oliver et al., 2007). These studies, and the data presented here, allude to the possibility of gene-environment interactions previously demonstrated for Rett and Smith-Magenis syndromes in which social motivation for challenging behaviours is part of the behavioural phenotype of the syndrome (Oliver, Murphy and Corbett, 1993; In review citation withheld for blind review).

Finally, the findings suggest adult eye contact (particularly from mother), and laughing and smiling are behaviours that individuals with Angelman syndrome find particularly reinforcing. These behaviours occur naturally and with high frequency in everyday life. The reinforcing properties of social interaction could be harnessed to develop more effective behavioural intervention programs for individuals with Angelman syndrome. Further work is needed to 
determine whether the difficult behaviours reported to be associated with Angelman syndrome such as aggression and attention seeking (Clarke et al., 2000; Clayton-Smith, 2001; Summers et al., 1995) are part of the same response class of approach behaviours with a function of gaining and maintaining social contact. Increased understanding of the function of any difficult behaviour could be used to develop behavioural strategies to reduce their occurrence. 


\section{References}

Aman, M. \& Singh, N. (1986). Abberant Behavior Checklist Manual. New York: Slosson Educational Publications, Inc.

Andersen, W. H., Rasmussen, R. K., \& Stromme, P. (2001). Levels of cognitive and linguistic development in Angelman syndrome: a study of 20 children. Logopedics, Phoniatrics, Vocology, 26, 29.

Angelman, H. (1965). "Puppet" children. A report of three cases. Developmental Medicine \& Child Neurology, 7, 681-688.

Berg, J. M. \& Pakula, Z. (1972). Angelman's ("happy puppet") syndrome. American Journal of Diseases of Children, 123, 72-74.

Cassidy, S. B., Dykens, E., \& Williams, C. A. (2000). Prader-Willi and Angelman syndromes: sister imprinted disorders. American Journal of Medical Genetics, 97, 136-146.

Clarke, D. J. \& Marston, G. (2000). Problem behaviors associated with 15q- Angelman syndrome. American Journal of Mental Retardation, 105, 25-31.

Clayton-Smith, J. (2001). Angelman Syndrome: Evolution of the phenotype in adolescents and adults. Developmental Medicine \& Child Neurology, 43, Jul-480.

Clayton-Smith, J. \& Laan, L. (2003). Angelman syndrome: a review of the clinical and genetic aspects. Journal of Medical Genetics, 40, 87-95.

Dooley, J. M., Berg, J. M., Pakula, Z., \& MacGregor, D. L. (1981). The puppet-like syndrome of Angelman. American Journal of Diseases of Children, 135, 621-624.

Fridman, C., Varela, M. C., Kok, F., Diament, A., \& Koiffmann, C. P. (2000). Paternal UPD15: further genetic and clinical studies in four Angelman syndrome patients. American Journal of Medical Genetics, 92, 322-327.

Haahr, M. (2002). Random sequence generator. http://www.random.org/sform.html [On-line].

Horsler, K. and Oliver, C. (2006a). The behavioural phenotype of Angelman syndrome. Journal of Intellectual Disability Research, 50, 33-53.

Horsler, K. and Oliver, C. (2006b). Environmental influences on the behavioural phenotype of Angelman syndrome. American Journal on Mental Retardation, 11, 311-321.

Jiang, Y., Lev-Lehman, E., Bressler, J., Tsai, T. F., \& Beaudet, A. L. (1999). Genetics of Angelman syndrome. American Journal of Human Genetics, 65, 1-6. 
Jolleff, N. \& Ryan, M. M. (1993). Communication development in Angelman's syndrome. Archives of Disease in Childhood, 69, 148-150.

Laan, L. A., Halley, D. J., den Boer, A. T., Hennekam, R. C., Renier, W. O., \& Brouwer, O. F. (1998). Angelman syndrome without detectable chromosome 15q11-13 anomaly: clinical study of familial and isolated cases. American Journal of Medical Genetics, 76, 262-268.

Lalli, J. S., Mace, F. C., Wohn, T., \& Livezey, K. (1995). Identification and modification of a responseclass hierarchy. Journal of Applied Behavior Analysis, 28, 551-559.

Leslie, J. C. (2002). The definition of reponse classes, response definition and response shaping. In J.C.Leslie (Ed.), Essential Behaviour Analysis (pp. 55-58). London: Arnold.

Lossie, A. C., Whitney, M. M., Amidon, D., Dong, H. J., Chen, P., Theriaque, D. et al. (2001). Distinct phenotypes distinguish the molecular classes of Angelman syndrome. Journal of Medical Genetics, 38, 834-845.

Martin, N., Oliver, C., \& Hall, S. (2002). ObsWin Version 3.0. Birmingham: The University of Birmingham.

Moncla, A., Malzac, P., Livet, M. O., Voelckel, M. A., Mancini, J., Delaroziere, J. C. et al. (1999a). Angelman syndrome resulting from UBE3A mutations in 14 patients from eight families: clinical manifestations and genetic counselling. Journal of Medical Genetics, 36, 554-560.

Moncla, A., Malzac, P., Voelckel, M. A., Auquier, P., Girardot, L., Mattei, M. G. et al. (1999b). Phenotype-genotype correlation in 20 deletion and 20 non-deletion Angelman syndrome patients. European Journal of Human Genetics, 7, 131-139.

Oliver, C., Demetriades, L., \& Hall, S. (2002). Effects of environmental events on smiling and laughing behavior in Angelman syndrome. American Journal of Mental Retardation, 107, 194-200.

Oliver, C., Horsler, K., Berg, K., Bellamy, G., Dick, K. \& Griffiths, E. (2007). Genomic imprinting and the expression of affect in Angelman syndrome. What's in the smile? Journal of Child Psychology and Psychiatry, 48, 571-579.

Oliver, C., Murphy, G., Crayton, L. \& Corbett, J.A. (1993). Self-injurious behaviour in Rett syndrome: Interactions between features of Rett syndrome and operant conditioning. Journal of Autism and Developmental Disorders, 23, 91-109.

Peters, S. U., Goddard-Finegold, J., Beaudet, A. L., Madduri, N., Turcich, M., \& Bacino, C. A. (2004). Cognitive and adaptive behavior profiles of children with Angelman syndrome. American Journal of Medical Genetics, Part A. 128, 110-113. 
Richman, D. M., Wacker, D. P., Asmus, J. M., Casey, S. D., \& Andelman, M. (1999). Further analysis of problem behavior in response class hierarchies. Journal of Applied Behavior Analysis, 32, 269-283.

Richman, D., Gernat, E., \& Teichman, H. (2006). The effects of social stimuli on smiling and laughing in young children with Angelman syndrome. American Journal on Mental Retardation, 111, 442-446.

Saitoh, S., Buiting, K., Cassidy, S. B., Conroy, J. M., Driscoll, D. J., Gabriel, J. M. et al. (1997). Clinical spectrum and molecular diagnosis of Angelman and Prader-Willi syndrome patients with an imprinting mutation. American Journal of Medical Genetics, 68, 195-206.

Sandanam, T., Beange, H., Robson, L., Woolnough, H., Buchholz, T., \& Smith, A. (1997). Manifestations in institutionalised adults with Angelman syndrome due to deletion. American Journal of Medical Genetics, 70, 415-420.

Sparrow, S., Balla, D., \& Cicchetti, V. (1984). Vineland Adaptive Behavior Scales. Circle Pines, Minneapolis: American Guidance Service.

Summers, J. A., Allison, D. B., Lynch, P. S., \& Sandler, L. (1995). Behaviour problems in Angelman syndrome. Journal of Intellectual Disability Research, 39, 97-106.

Summers, J. A. \& Feldman, M. A. (1999). Distinctive pattern of behavioral functioning in Angelman syndrome. American Journal of Mental Retardation, 104, 376-384.

Williams, C. A., Angelman, H., Clayton-Smith, J., Driscoll, D. J., Hendrickson, J. E., Knoll, J. H. et al. (1995). Angelman syndrome: consensus for diagnostic criteria.Angelman Syndrome Foundation. American Journal of Medical Genetics, 56, 237-238.

Williams, C. A. \& Frias, J. L. (1982). The Angelman ("happy puppet") syndrome. American Journal of Medical Genetics, 11, 453-460.

Zori, R. T., Hendrickson, J., Woolven, S., Whidden, E. M., Gray, B., \& Williams, C. A. (1992). Angelman syndrome: clinical profile. Journal of Child Neurology, 7, 270-280.

Table 1. Demographic information on the participants

\begin{tabular}{cccccc}
\hline Participant & Gender & $\begin{array}{c}\text { Chronological } \\
\text { Age (Years) }\end{array}$ & Genetic cause & $\begin{array}{c}\text { Adaptive } \\
\text { behavior } \\
\text { composite } \\
\text { (months) }\end{array}$ & $\begin{array}{c}\text { Adaptive } \\
\text { deficit }^{{ }^{2}}\end{array}$ \\
\hline \hline 1 & female & 3.0 & Deletion & 10.5 & Moderate \\
2 & male & 3.3 & Deletion & 12.0 & Moderate \\
3 & male & 3.8 & Deletion & 10.3 & Moderate \\
4 & female & 4.7 & Imprinting defect & 17.8 & Moderate
\end{tabular}




\begin{tabular}{ccclcc}
5 & female & 5.3 & Deletion & 12.3 & Severe \\
6 & male & 6.6 & Deletion & 10.3 & Severe \\
7 & female & 6.6 & Deletion & 8.0 & Severe \\
8 & male & 7.3 & Deletion & 10.0 & Severe \\
9 & male & 8.1 & Deletion & 12.0 & Severe \\
10 & female & 8.4 & Deletion & 19.0 & Severe \\
11 & female & 9.6 & Deletion & 16.0 & Severe \\
12 & male & 10.3 & Deletion & 15.3 & Profound \\
13 & male & 12.1 & Deletion & 10.3 & Profound \\
14 & female & 18.3 & UPD & 20.7 & Profound \\
15 & male & 18.5 & Deletion & 20.7 & Profound \\
\hline
\end{tabular}

${ }^{1}$ Mean age equivalent derived from the Vineland Adaptive Behavior Scale

${ }^{2}$ Adaptive level derived from Vineland Adaptive Behavior Scale 
Table 2. Experimental conditions employed during observations

\begin{tabular}{ll}
\hline \multicolumn{1}{c}{ Condition } & \multicolumn{1}{c}{ Adult behaviour } \\
\hline \hline $\begin{array}{l}\text { Eye contact } \\
\text { and talking }\end{array}$ & $\begin{array}{l}\text { Maintain eye contact with child. Talk naturally to the child about activities } \\
\text { of the last week, plans for the day etc. It does not matter if the talk is } \\
\text { repeated. Do not make demands on the child by asking them to do } \\
\text { anything. Do not laugh or smile. }\end{array}$ \\
\hline $\begin{array}{l}\text { Eye contact, } \\
\text { no talking }\end{array}$ & Maintain eye contact. Do not make any noises. Do not laugh or smile. \\
\hline $\begin{array}{l}\text { No eye } \\
\text { contact and } \\
\text { talking }\end{array}$ & $\begin{array}{l}\text { Look down towards floor. Talk naturally to the child about activities of the } \\
\text { last week, plans for the day etc. It does not matter if the talk is repeated. } \\
\text { Do not make demands on the child by asking them to do anything. Do not }\end{array}$ \\
\hline $\begin{array}{l}\text { No eye } \\
\text { lantact, no } \\
\text { talking }\end{array}$ & Look down towards floor. Do not make any noises. Do not laugh or smile. \\
\hline
\end{tabular}


Table 0.2 Definitions and reliability indices for participant behaviours

\begin{tabular}{lll}
\hline \multicolumn{1}{c}{$\begin{array}{c}\text { Adult } \\
\text { behaviours }\end{array}$} & Definition & Kappa \\
\hline \hline Eye contact & Face turned towards that of the child and eyes directed towards the face of the child & .53 \\
\hline \hline Talking & Any verbal action & .50 \\
\hline \hline $\begin{array}{l}\text { Laughing / } \\
\text { smiling }\end{array}$ & $\begin{array}{l}\text { Any horizontal stretching of the lips, upturning of the corners of the mouth followed } \\
\text { by parting of the lips and viewing of the teeth, or any short bust of inarticulate voiced } \\
\text { noises accompanied by an opening of the mouth, upturning corners of the lips, } \\
\text { displayed teeth, half or completely shut eyes and raised cheeks. }\end{array}$ \\
\hline \hline Touching & $\begin{array}{l}\text { Physical contact with any part of the child's body as a result of moving part of their } \\
\text { body towards that of the child. If adult is already in physical contact with a body part } \\
\text { of the child, the adult moving the part of the body of the child in anyway. }\end{array}$ \\
\hline \hline
\end{tabular}

\section{Participant} Behaviours

Looking at Face turned towards that of the adult and eyes directed towards the face of the adult adult Looking at camera Laughing / smiling

Reaching for adult Touching adult head

Touching adult body

Pulling adult head

Pulling adult body

\section{Definition}

Kappa

Face turned towards the camera and eyes directed towards the camera

Any horizontal stretching of the lips, upturning of the corners of the mouth followed by parting of the lips and viewing of the teeth, or any short bust of inarticulate voiced noises accompanied by an opening of the mouth, upturning corners of the lips, displayed teeth, half or completely shut eyes and raised cheeks.

Moving arms towards the body or head of the adult but not able to make contact with the adult

Touching any part of the adult on neck or above as a result of moving part of their body towards the adult. If child is already touching a body part of the adult above the neck, the child moving that part of the body of the adult in a gentle non-aggressive way. Does not include movements of legs or feet.

Touching any part of the adult below their neck as a result of moving part of their body towards the adult. If child is already touching a body part of the adult below the neck, the child moving that part of the body of the adult in a gentle non-aggressive way. Does not include movements of legs or feet.

Child has physical contact with the adult's neck or above and grasps and pulls the adult with force and in an aggressive manner. Includes pulling head, face, hair.

Child has physical contact with part of the adult below the neck and grasps and pulls the adult with force and in an aggressive manner. Includes pulling jumpers, arms, legs.
.75

.85

.76

.84

.75

.81

.77 
Table 3 Mean, SD and range of occurrence of adult laughing/smiling and touching across conditions

\begin{tabular}{llcccccc} 
& & \multicolumn{3}{c}{ Adult laugh/smile } & \multicolumn{3}{c}{ Adult touch } \\
\cline { 3 - 8 } Person & Condition & $\begin{array}{c}\text { Mean \% } \\
\text { intervals }\end{array}$ & SD & range & $\begin{array}{c}\text { Mean \% } \\
\text { intervals }\end{array}$ & SD & range \\
& & 16.34 & 16.73 & $0-55$ & 27.91 & 28.26 & $0-81$ \\
\hline \hline Familiar & Adult eye contact, talking & 5.25 & 5.98 & $0-16$ & 12.50 & 21.61 & $0-75$ \\
& Adult eye contact, not talking & 5.66 & 10.27 & $0-31$ & 11.78 & 15.44 & $0-53$ \\
& Adult not eye contact, talking & 8.69 & & & & \\
& Adult no eye contact, not & 3.41 & 7.09 & $0-23$ & 6.25 & 8.09 & $0-23$ \\
& talking & & & & & & \\
\hline Unfamiliar & Adult eye contact, talking & 7.10 & 8.07 & $0-20$ & 8.95 & 12.79 & $0-31$ \\
& Adult eye contact, not talking & 4.55 & 4.86 & $0-16$ & 10.31 & 14.39 & $0-43$ \\
& Adult no eye contact, talking & 2.98 & 6.03 & $0-17$ & 12.30 & 20.41 & $0-61$ \\
& Adult no eye contact, not & .85 & 1.46 & $0-3$ & 4.78 & 6.46 & $0-17$ \\
& talking & & & & & & \\
\hline
\end{tabular}


Table 4. Percentage of time each participant showed laugh/smile, looking and approach behaviours across all experimental sessions

Percentage of intervals

\begin{tabular}{ccccc}
\hline Participant & Laugh/smile & Look at adult & Look at camera & Approach adult \\
\hline \hline 1 & 15.23 & 34.77 & 8.40 & 26.17 \\
2 & 44.84 & 61.14 & 10.33 & 42.66 \\
3 & 6.05 & 33.20 & 7.23 & 29.69 \\
6 & 14.29 & 24.75 & 5.03 & 22.18 \\
7 & 27.54 & 35.94 & 16.41 & 43.36 \\
8 & 24.98 & 57.62 & 19.92 & 9.18 \\
9 & 39.45 & 65.04 & 6.25 & 30.39 \\
10 & 71.88 & 78.91 & 6.45 & 42.58 \\
11 & 32.81 & 84.77 & 15.04 & 25.59 \\
12 & 11.52 & 54.10 & 9.77 & 20.70 \\
15 & 75.39 & 66.21 & 24.02 & 16.60 \\
\hline \hline Mean & 33.18 & 54.23 & 11.71 & 28.10 \\
SD & 23.29 & 19.71 & 6.25 & 11.19 \\
\hline
\end{tabular}


Figure 1. Mean duration of laughing and smiling (upper panels), looking at adults (middle panels) and approach behaviours (lower panels) shown by children with Angelman syndrome in conditions in which adult familiarity, eye contact and talking were manipulated.

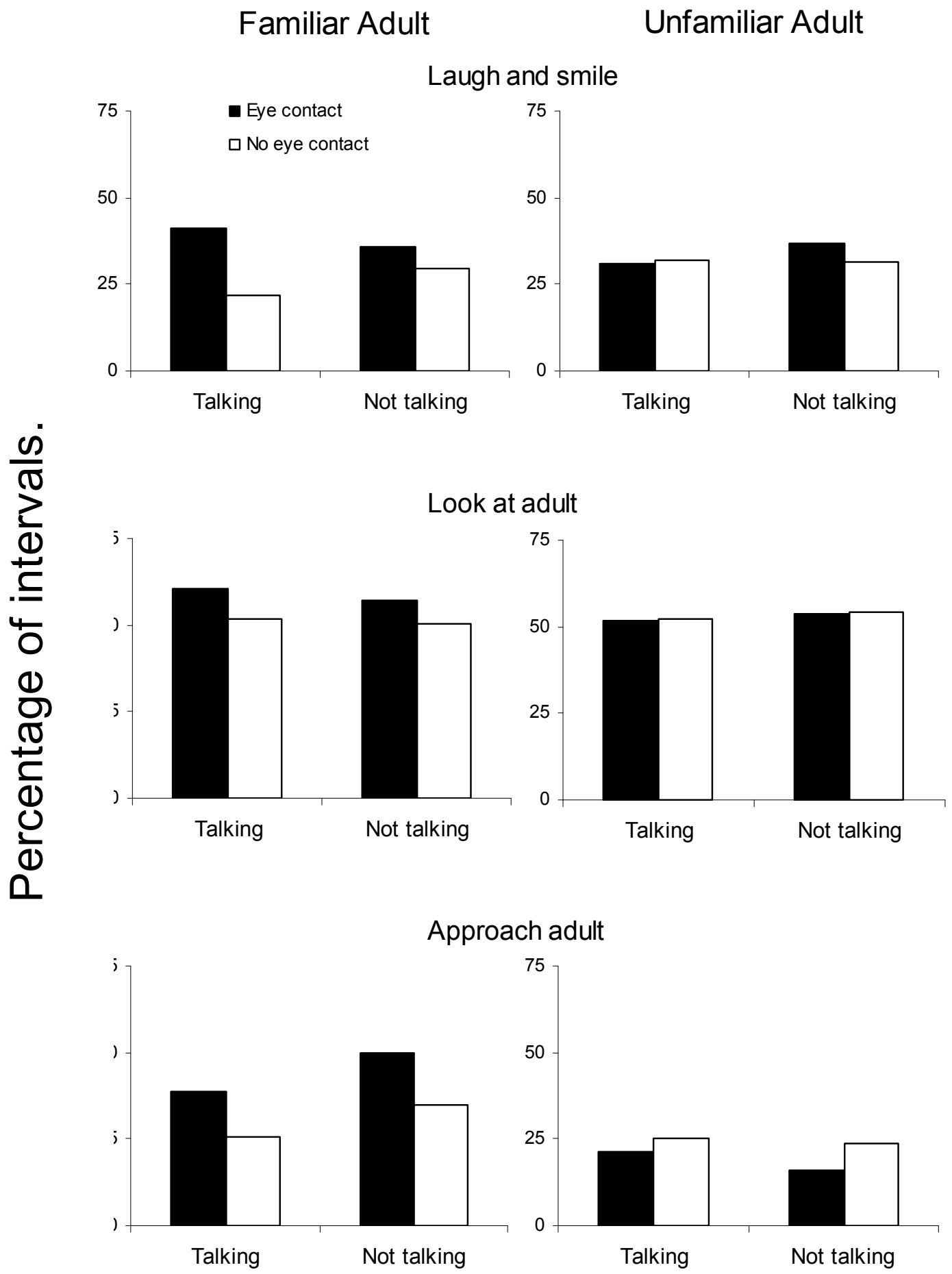

